\title{
HALO PROPERTIES IN COSMOLOGICAL SIMULATIONS OF SELF-INTERACTING COLD DARK MATTER
}

\author{
Romeel Davé ${ }^{1,3}$, David N. Spergel ${ }^{1}$, Paul J. Steinhardt ${ }^{2}$, Benjamin D. Wandelt ${ }^{2}$ \\ Draft version June 3, 2018
}

\begin{abstract}
We present a comparison of halo properties in cosmological simulations of collisionless cold dark matter (CDM) and self-interacting dark matter (SIDM) for a range of dark matter cross sections. We find, in agreement with various authors, that CDM yields cuspy halos that are too centrally concentrated as compared to observations. Conversely, SIDM simulations using a Monte Carlo $N$-body technique produce halos with significantly reduced central densities and flatter cores with increasing cross section. We introduce a concentration parameter based on enclosed mass that we expect will be straightforward to determine observationally, unlike that of Navarro, Frenk \& White, and provide predictions for SIDM and CDM. SIDM also produces more spherical halos than CDM, providing possibly the strongest observational test of SIDM. We discuss our findings in relation to various relevant observations as well as SIDM simulations of other groups. Taking proper account of simulation limitations, we find that a dark matter cross section per unit mass of $\sigma_{\mathrm{DM}} \approx 10^{-23}-10^{-24} \mathrm{~cm}^{2} \mathrm{GeV}^{-1}$ is consistent with all current observational constraints.
\end{abstract}

Subject headings: Dark matter, galaxies: formation, galaxies: halos, methods: n-body simulations

\section{INTRODUCTION}

The cold dark matter (CDM) family of cosmological models provides an excellent description of a wide vari-

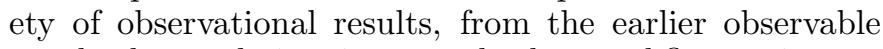
epochs detected via microwave background fluctuations to present-day observations of galaxies and large-scale structure. A "concordance model" with roughly one-third matter and two-thirds vacuum energy, either a cosmological constant or quintessence (Caldwell, Dave \& Steinhardt 1998), is consistent with almost all current observations on scales $\gtrsim 1$ Mpc (Bahcall et al. 1999).

Recently, improving observations and numerical techniques have enabled a comparison of CDM scenarios to observations on galactic scales of $\sim$ few kpc. The results have not been encouraging. There are a number of distinct observations that may be in conflict with predictions of CDM:

- The density profile of galaxies in the inner few kiloparsecs appears to be much shallower than predicted by numerical simulations (Navarro, Frenk \& White 1996, hereafter NFW). For density profiles characterized by $\rho(r) \propto r^{\alpha}$ as $r \rightarrow 0$, CDM predicts $\alpha \approx-1.5$ with little scatter (Moore et al. 1999), while current $\mathrm{H} \alpha$ observations suggest $\alpha \approx-0.5$ with significant scatter (Swaters, Madore \& Trewhella 2000; Dalcanton \& Bernstein 2000, though see van den Bosch \& Swaters 2000).

- The central density of dark matter halos is observed to be $\rho_{c} \sim 0.02 M_{\odot} \mathrm{pc}^{-3}$ roughly independent of halo mass (Firmani et al. 2000b), while CDM predicts halos with $\rho_{c} \gtrsim 1 M_{\odot} \mathrm{pc}^{-3}$ at dwarf galaxy masses, increasing to larger masses (Moore et al. 1999).

${ }^{1}$ Princeton University Observatory, Princeton, NJ 08544

${ }^{2}$ Department of Physics, Princeton University, Princeton, NJ 08544

${ }^{3}$ Spitzer Fellow
- The number of dwarf galaxies in the Local Group is an order of magnitude fewer than predicted by CDM simulations, with the discrepancy growing towards smaller masses (Moore et al. 1999a; Klypin et al. 1999).

- Hydrodynamic simulations produce galaxy disks that are too small and have too little angular momentum, yielding a Tully-Fisher relation whose zeropoint is off by several magnitudes from observations (Navarro \& Steinmetz 2000).

- The robustness of rapidly rotating bars in high surface brightness spiral galaxies implies lower density cores than predicted by CDM (de Battista \& Sellwood 1998).

- Cluster CL 0024+1654 is nearly spherical with a large, soft core, while CDM typically predicts triaxial clusters with cuspy cores (Tyson, Kochanski \& Dell'antonio 1998, though see Miralda-Escudé 2000 for a counterexample).

Each piece of evidence taken individually is perhaps not convincing enough to claim that CDM has failed on galactic scales. For instance, until recently there was controversy amongst simulators regarding inner profiles (Kravtsov et al. 1998), but more careful simulations have converged on a consistent prediction (Klypin 2000). Observationally, inner galactic profiles are uncertain due to beam smearing effects in $\mathrm{H}$ I observations (Swaters. Madore \& Trewhella 2000; van den Bosch et al. 2000), though samples of high-resolution $\mathrm{H} \alpha$ observations continue to show shallower profiles than predicted by CDM (Dalcanton \& Bernstein 2000). The number of observed Local Group dwarf galaxies may be reconciled with CDM 
via plausible scenarios for suppressed galaxy formation (e.g. Bullock, Kravtsov \& Weinberg 2000), or else compact high-velocity clouds could represent the "missing satellites" that are seen in $N$-body simulations (Blitz et al. 1999] Hydrodynamic simulations of disk galaxy formation are fraught with the usual concerns about the effects of feedback, artificial viscosity and resolution, though it appears the discrepancies above are due to the underlying dark matter distribution (Navarro \& Steinmetz 2000).

It becomes more interesting to consider alternatives to conventional CDM when one recognizes that all these discrepancies may be symptomatic of a single cause: Dark matter halos in CDM simulations appear to be more centrally concentrated than observed. Recognizing this, various authors have recently forwarded a plethora of alternative dark matter theories that suppress the central concentration of dark matter in galaxy halos. Among such theories are that the dark matter is warm (SommerLarse 1 \& Dolgov 2000; Colin, Avila-Reese \& Valenzuela 2000 Hannestad \& Scherrer 2000), repulsive (Goodman 2000 fluid (Peebles 2000), fuzzy (Hu, Barkana \& Gruzinov 2000), decaying (Cen 2000), annihilating (Kaplinghat, Knox \& Turner 2000), and the alternative we investigate here, self-interacting (SIDM; Spergel \& Steinhardt 2000). Interestingly, all theories may be tuned to solve the problems mentioned above (at least in analytic approximations), all theories may be motivated from particle physics considerations, and all theories retain the desirable properties of CDM on extragalactic scales (though warm dark matter is non-trivially constrained by this requirement; see Narayanan et al. 2000).

SIDM is governed by a single free parameter, the cross section per unit mass $\sigma_{\mathrm{DM}}$ of the interacting dark matter particle. Spergel \& Steinhardt suggested $\sigma_{\mathrm{DM}} \sim$ $10^{-22}-10^{-25} \mathrm{~cm}^{2} \mathrm{GeV}^{-1}$ in order to reduce the central concentration of galaxy halos by a sufficient amount to alleviate the above problems. Intriguingly, this value is close to the cross section of ordinary hadrons, motivating some particular particle physics candidates for SIDM (Steinhardt et al., in preparation). If $\sigma_{\mathrm{DM}}$ is significantly smaller than this range, then the optical depth at galactic densities is much less than unity, implying that SIDM would have a negligible effect on the dark matter distribution in halos.

A qualitative picture of the evolution of an SIDM halo is as follows: At early times there is no difference between SIDM and CDM since the densities and peculiar velocities are sufficiently low that collisions are rare; hence SIDM makes identical predictions to CDM regarding cosmic microwave background fluctuations and the Lyman alpha forest. As the halo forms and grows via gravitational instability, the central density increases. Eventually, collisions are so frequent that dark matter particles scatter out of the center as fast as they are accreted, and the density growth is halted, forming a core. Such a limit is not present in the CDM model, where the central density grows unchecked. The SIDM core then begins to extend while retaining constant central density. Heat transfer from the outer parts of the halo raises the temperature in the halo core. If the halo is truly isolated, then eventually the core thermalizes with the exterior resulting in an isothermal halo with a steep density profile. This initiates gravothermal collapse, where the direction of heat transfer is reversed and the exterior begins to cool the halo center. However, in a realistic cosmological setting, galaxies constantly accrete material, keeping the outer halo hot and heat flowing inwards, thus delaying core collapse. The interplay between collisional heat transfer and accretion determines whether a halo will undergo core collapse in a Hubble time.

It is important to appreciate that the transport behavior does not change monotonically with $\sigma_{\text {DM }}$. For small cross-sections, heat transfer increases with $\sigma_{\mathrm{DM}}$ since the frequency of collisions increases; however, for large crosssections, the conductivity $\kappa \propto \sigma_{\mathrm{DM}}^{-1} \rightarrow 0$ and no heat transfer occurs. Thus, as we discuss in $\$ 6$, the fluid approximation is a poor decription of SIDM in the moderate cross-section regime proposed by Spergel and Steinhardt. Furthermore, the behavior in the moderate cross-section regime cannot be surmised by interpolating between the fluid and the non-interacting CDM regimes. A proper treatment of the SIDM proposal, which includes the interplay of accretion and heat transfer, its non-monotonic dependence $\sigma_{\mathrm{DM}}$, and the effects of merging demands numerical simulations designed to explore the moderate crosssection regime.

In this paper we investigate the statistical properties of halos in SIDM and CDM in cosmological N-body simulations. Our spatial and mass resolutions are sufficient to probe the inner regions $\left(\sim 1 h^{-1} \mathrm{kpc}\right)$ of small halos ( $\gtrsim$ few $\times 10^{9} M_{\odot}$ ), while maintaining sufficient volume so as to have a significant sample of such halos. We use a Monte Carlo technique similar to Kochanek \& White (2000) to model collisions. The primary difference between our simulations and prior investigations (discussed in more detail in $8_{6}$ ) is that we model a cosmologically-significant random volume of the universe with self-interaction cross sections in the range favored by Spergel \& Steinhardt, enabling us to characterize the statistical properties of halos as we vary $\sigma_{\mathrm{DM}}$.

$\$ 2$ describes our initial conditions and simulation techniques using a Monte Carlo $N$-body approach. In $\$ 3$ we compare the structural properties of halos in CDM versus SIDM models with several cross sections. In particular we examine their central densities, inner profile slopes $\alpha$, the mass dependence of $\alpha$, concentrations, phase space densities, and ellipticities, and where possible compare to observations. In $\$$ we use lower resolution simulations to test the effects of finite particle numbers in our Monte Carlo method. In $\$$ we examine the subhalo population around the largest halo in our simulations. In $\$ 6$ we compare our findings to the simulations of other groups who have conducted numerical studies of self-interacting dark matter, and examine results from a wider range of $\sigma_{\mathrm{DM}}$. We summarize our results and discuss observational constraints in $\$ \sqrt{7}$. We find that SIDM with $\sigma_{\mathrm{DM}} \approx 10^{-23}-10^{-24} \mathrm{~cm}^{2} \mathrm{GeV}^{-1}$ produces halos that are in better agreement than collisionless CDM for a wide variety of observations.

\section{SIMULATING SIDM}

\subsection{Code and Cosmology}

We use a modified version of GADGET Springel, Yoshida \& White 2000), a publically-available TreeSPH code for distributed-memory parallel machines. Here we 
only employ the gravitational $N$-body portion. We evolve a $4 h^{-1} \mathrm{Mpc}$ randomly-chosen volume of a $\Lambda \mathrm{CDM}$ universe with $\Omega=0.3, \Omega_{\Lambda}=0.7, H_{0}=70 \mathrm{~km} \mathrm{~s}^{-1} \mathrm{Mpc}^{-1}$, and $\sigma_{8}=0.8$, similar to the "concordance model" in agreement with a wide variety of observations Bahcall et al. 1999 We generate initial conditions using COSMICS (Ma $\&$ Beitschinger 1995) at $z=49.7$, where our particle distribution first becomes nonlinear, and evolve to $z=0$. We employ $128^{3}$ dark matter particles in each run, resulting in a dark matter particle mass of $m_{p}=3.6 \times 10^{6} M_{\odot}$, and a spline kernel softening of $\epsilon=1 h^{-1} \mathrm{kpc}$ (i.e. force is Newtonian at $2 \epsilon)$. To test resolution effects, we also run a suite of simulations with $64^{3}$ particles and $\epsilon=2 h^{-1} \mathrm{kpc}$. Their initial conditions have an initial density field identical to the $128^{3}$ runs, constructed by sampling at alternate grid points.

While our $4 h^{-1} \mathrm{Mpc}$ box is small, well below the nonlinear scale at $z=0$, we are interested here in the behavior on scales of a few kpcs, and it is unlikely that the missing large-scale power would have a significant effect on the inner portions of halos. In addition, our primarily conclusions are based on a comparative study between collisionless and collisional dark matter for individual halos, so we expect these results to be robust to volume effects.

\subsection{Modeling self-interactions}

We have modified GADGET to include self-interactions using a Monte Carlo $N$-body technique to probabilistically incorporate collisions, along the same lines as Burkert (2000) and Kochanek \& White, closer to the latter as we use $\Delta \mathbf{v}$ from individual particles colliding rather than setting $\Delta \mathbf{v}$ to be the particle's velocity; see the discussion in Kochanek \& White. Each pair of particles with positions and velocities $\left(\mathbf{r}_{1}, \mathbf{v}_{1}\right)$ and $\left(\mathbf{r}_{2}, \mathbf{v}_{2}\right)$, separated by $\delta x \equiv\left|\mathbf{r}_{1}-\mathbf{r}_{2}\right| /(2 \epsilon)$ and $\delta v \equiv\left|\mathbf{v}_{1}-\mathbf{v}_{2}\right|$, interact with a probability given by

$$
P=f_{\text {geom }}(\delta x) \frac{\delta v \Delta t}{\lambda_{X}},
$$

where $\Delta t$ is the timestep,

$$
\lambda_{X}=\frac{4 \pi(2 \epsilon)^{3}}{3 m_{p}} \frac{1}{\sigma_{\mathrm{DM}}}
$$

and

$$
f_{\text {geom }}(\delta x)=N \frac{\int_{0}^{1} W(\delta x) W\left(\delta x+\delta x^{\prime}\right) d\left(\delta x^{\prime}\right)}{\int_{0}^{1} W^{2}\left(\delta x^{\prime}\right) d\left(\delta x^{\prime}\right)},
$$

where $W$ is the cubic spline kernel used in GADGET. This geometrical factor weights the probability of interaction by the product of spline kernel-weighted density distributions of the two particles at their given separation. The normalization $N$ is set by requiring that

$$
\int_{0}^{1} f_{\text {geom }}(\delta x) 4 \pi \delta x^{2} d(\delta x)=1,
$$

which ensures that when a particle has interacted with all its neighbors within $2 \epsilon$, the resulting probability is equivalent to

$$
P=\sigma_{\mathrm{DM}} \rho \delta v \Delta t,
$$

where $\rho$ is the local dark matter density.

In our code, the scatterings are performed between individual particles at the time that the acceleration between those particles is being computed (i.e. during the "treewalk"). In order to ensure that all possible scatterings are considered, a tree cell is opened whenever it is within $2 \epsilon$ of a particle, regardless of the opening criterion.

If two particles scatter, their velocities are randomly reoriented, keeping the magnitudes of their velocities fixed. In practice, a running sum is kept of the change in velocity due to the interactions that a given particle undergoes on every processor, and at the end of the step the velocity change for each particle is summed over all processors and added to that particle's velocity. In this way, energy and momentum are explicitly conserved, even if the scattered particles are on different processors, or a particle undergoes more than one scatter in a single timestep (which is very rare for the cross sections considered here).

We consider $\sigma_{\mathrm{DM}}=0$ (collisionless), $10^{-24}$, and $10^{-23} \mathrm{~cm}^{2} \mathrm{GeV}^{-1}$. We also examined $\sigma_{\mathrm{DM}}=$ $10^{-25} \mathrm{~cm}^{2} \mathrm{GeV}^{-1}$ and $\sigma_{\mathrm{DM}}=10^{-22} \mathrm{~cm}^{2} \mathrm{GeV}^{-1}$ in a $64^{3}$ simulation, which we will examine in $\$$. The total number of collisions per particle in our simulations are 1.01 for $\sigma_{\mathrm{DM}}=10^{-24} \mathrm{~cm}^{2} \mathrm{GeV}^{-1}$, and 6.05 for $\sigma_{\mathrm{DM}}=$ $10^{-23} \mathrm{~cm}^{2} \mathrm{GeV}^{-1}$, with slightly lower numbers (0.9 and 5.3 ) for the $64^{3}$ runs. Note that a factor of ten increase in $\sigma_{\mathrm{DM}}$ translates only to a factor of six increase in the number of collisions, since the lowered central densities (\$3.2) partially compensates for the increase in $\sigma_{\mathrm{DM}}$. All runs were performed on Fluffy, a 32-processor Beowulfclass machine at Princeton, with each $128^{3}$ run taking approximately one week.

\subsection{The Simulated Halo Sample}

We identify dark matter halos using SKID ${ }^{1}$ (Spline Kernel Interpolative DENMAX; see Katz, Weinberg \& Hernquist 1996), with a linking length of $2 \epsilon$. We only consider halos containing 64 or more particles, to ensure a roughly complete sample of such halos in our simulations (Weinberg et al. 1999). Table 1 lists the number of halos for identified in these simulations.

A specific resolution issue arises from the finite number of particles used to probabalistically model collisions in the SIDM simulations: The number of particles in a given halo must be high enough to properly Monte Carlo sample the distribution. As we will show in $\S$, halos with $\gtrsim 1000$ particles at $z=0$ seem to be accurately represented with this technique for the simulations considered here. This is quite restrictive, but still permits a significant sample of halos (roughly 30 in each $128^{3}$ run) with which to compute statistics. We also use the full sample of halos to examine certain aspects, but we will be cautious about interpretations made from halos below this "Monte Carlo resolution limit".

\section{HALO STRUCTURE}

\subsection{Halo Profiles}

We determine halo profiles $\rho(r)$ by spherical averages over radii $r=\epsilon \rightarrow 30 \epsilon$, in 20 equal intervals of $\log r$. 
TABLE 1

Simulation RESUlts.

\begin{tabular}{lc|cccc|cccc}
\hline \hline Model & \multirow{2}{*}{$\sigma_{\mathrm{DM}}\left(\mathrm{cm}^{2} \mathrm{GeV}^{-1}\right)$} & \multicolumn{3}{c}{$128^{3 *}$} \\
\hline & & $N_{\text {halo }}$ & $\alpha_{\text {med }}$ & $\rho_{c, \text { med }}{ }^{\ddagger}$ & $c_{M, \text { med }}$ & $N_{\text {halo }}$ & $\alpha_{\text {med }}$ & $\rho_{c, \text { med }}{ }^{\ddagger}$ & $c_{M, \text { med }}$ \\
\cline { 3 - 10 } CDM & 0 & 670 & -1.49 & 1.95 & 8.0 & 111 & -1.58 & 1.91 & 6.7 \\
SIDM & $10^{-24}$ & 647 & -0.93 & 0.22 & 5.6 & 106 & -1.44 & 0.36 & 4.8 \\
SIDM & $10^{-23}$ & 566 & -0.37 & 0.030 & 2.6 & 89 & -0.53 & 0.027 & 1.8 \\
Observed & & & $\approx-0.5$ & $\approx 0.02$ & - & & $\approx-0.5$ & $\approx 0.02$ & \\
\hline
\end{tabular}

${ }^{*} 128^{3}$ median values computed for all halos with $>1000$ particles ( $~ 30$ per run).

$\dagger 64^{3}$ median values computed for all halos with $>500$ particles $(\sim 10$ per run).

${ }^{\ddagger}$ Central dark matter density in $M_{\odot} \mathrm{pc}^{-3}$; values shown are extrapolated from $1 h^{-1} \rightarrow 0.5 \mathrm{kpc}$ using $\alpha_{\text {median }}$.

A sample of 16 halo profiles from our $128^{3}$ simulations is shown in Figure 1. Each panel shows a halo profile for $\sigma_{\mathrm{DM}}=0$ (solid), $10^{-24}$ (short dashed), and $10^{-23} \mathrm{~cm}^{2} \mathrm{GeV}^{-1}$ (long dashed). Dotted line segments emanating from the innermost radius point of the $\sigma_{\mathrm{DM}}=0$ curve indicate slopes $\alpha=-0.5,-1$ and -1.5 for comparison. The same corresponding halos are chosen from each simulation, allowing a case-by-case comparison of the effect of SIDM. The outer halo profiles $\left(r \gtrsim 10 h^{-1} \mathrm{kpc}\right)$ are virtually identical for each halo, showing that the effects of self-interactions are limited to the inner few kpcs of halos, and confirming that the same halos are being compared in the different simulations. The halos in the leftmost column are the four most massive ones in our simulations, while the halos in other columns are chosen randomly from a descending range of masses. Note that rightmost column shows halos with roughly 300 particles each, below our Monte Carlo resolution requirement of $\gtrsim 1000$ particles (i.e. $\gtrsim 3.6 \times 10^{9} M_{\odot}$ ), thus the effects of self-interactions are not necessarily accurately represented in these cases.

From Figure 1 it is immediately evident that SIDM produces halos that have enlarged central cores and shallower inner profiles. CDM halos are almost all cuspy $(\alpha \lesssim-1$ typically), while most $\sigma_{\mathrm{DM}}=10^{-23} \mathrm{~cm}^{2} \mathrm{GeV}^{-1}$ cores are close to flat. $\sigma_{\mathrm{DM}}=10^{-24} \mathrm{~cm}^{2} \mathrm{GeV}^{-1}$ leads to profiles that are intermediate between these two. In some cases, non-cuspy CDM halos are seen, especially at lower masses. In these cases the halo may have undergone recent merging activity that temporarily lowers the central density, which is particularly effective in smaller mass halos. Additionally, recent mergers that have not relaxed make it difficult to unambiguously identify the halo center about which to compute profiles, typically making profiles appear shallower. We make no cut in regards to the merging history or "isolatedness" of halos, but we do note that the missing large-scale power in our simulations will tend to generate fewer mergers, and make the largest objects in our simulations appear more isolated.

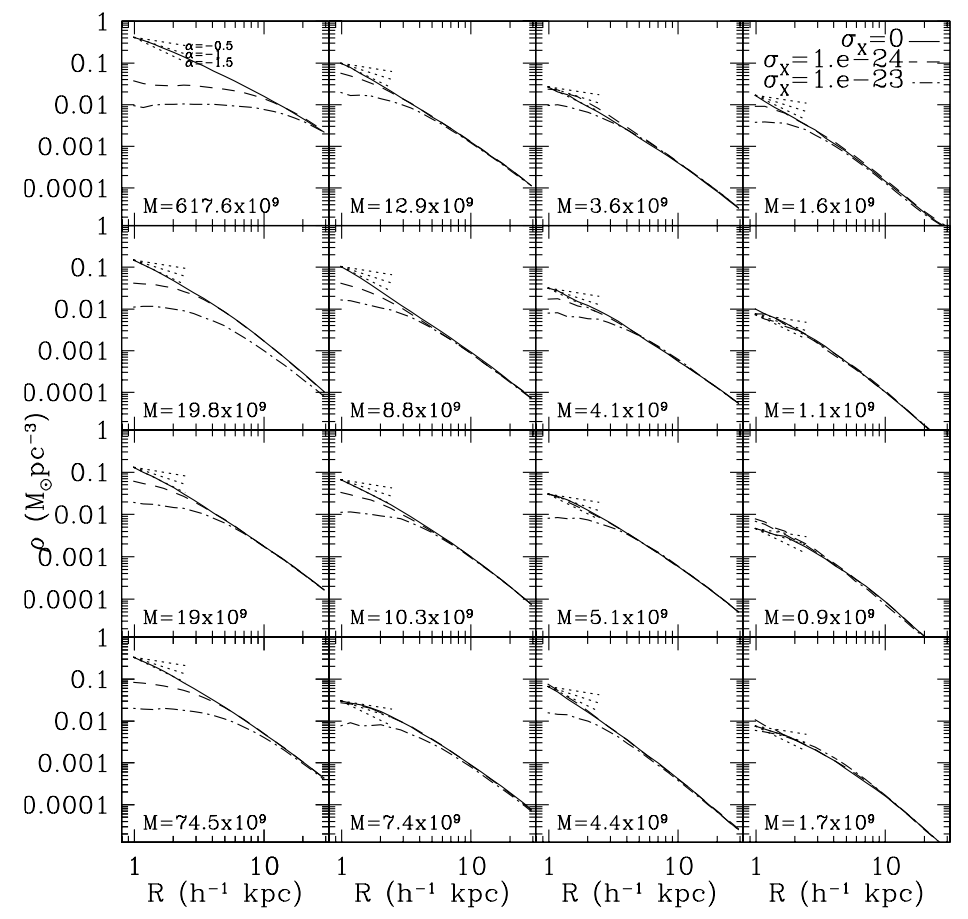

Figure 1: 16 selected halo profiles for collisionless (solid), $10^{-24} \mathrm{~cm}^{2} \mathrm{GeV}^{-1}$ (dashed), and $10^{-23} \mathrm{~cm}^{2} \mathrm{GeV}^{-1}$ (dotdashed). Corresponding halos from each simulation are presented, allowing a direct comparison of the effect of SIDM on a halo-by-halo basis. The total halo mass in $M_{\odot}$ for the $\sigma_{\mathrm{DM}}=0$ halo is shown in the lower right; the SIDM halo masses are typically within $20 \%$. The columns are ordered by mass, with the four highest mass halos shown in the leftmost column. Dotted lines from innermost point show reference slopes of $\alpha=-0.5,-1.0,-1.5$.

At high masses, the effect of SIDM is very prominent. The upper leftmost halo is Milky Way-sized $\left(6 \times 10^{11} M_{\odot}\right)$, and shows a large core of $\sim 15 h^{-1} \mathrm{kpc}$ for $\sigma_{\mathrm{DM}}=10^{-23} \mathrm{~cm}^{2} \mathrm{GeV}^{-1}\left(\sim 8 h^{-1} \mathrm{kpc}\right.$ for $\sigma_{\mathrm{DM}}=$ $\left.10^{-24} \mathrm{~cm}^{2} \mathrm{GeV}^{-1}\right)$. The core size and difference in inner slope become less prominent to lower masses, though this could be due to the increasing effects of unrelaxed halos, as well as the Monte Carlo resolution issues discussed earlier. 
We examine these issues quantitatively in $\$ 3.4$.

No evidence is seen for SIDM halo profiles that are isothermal, as would be expected if the cross section was so large that core collapse would occur on timescales significantly shorter than a Hubble time. This supports the analytic estimates of Spergel \& Steinhardt that core collapse on a Hubble time would not occur until $\sigma_{\mathrm{DM}} \gtrsim$ $10^{-22} \mathrm{~cm}^{2} \mathrm{GeV}^{-1}$.

Overall, there is a clear trend on a case-by-case basis that SIDM results in a reduced central density and shallower inner slope of the dark matter halo, with increasing $\sigma_{\mathrm{DM}}$ having a greater such effect.

\subsection{Central Densities}

Figure 2 shows the central density of dark matter halos $\rho_{c}$, taken to be the density at our innermost resolved radius $\epsilon$, as a function of halo mass. Here we only consider halos with more than 1000 particles, where our Monte Carlo technique has sufficient numbers to represent the collisional behavior (as we will discuss in \$4). The central halo density of galaxies is observed to be $\sim 0.02 M_{\odot} \mathrm{pc}^{-3}$ (Firmani et al. 2000b), and is consistent with being independent of halo mass. The observed range of halo densities is shown as the hatched region, with a majority of their data falling towards the lower end of that region. The arrow in the upper left indicates the increase in $\rho_{c}$ projecting the profile from $1 h^{-1} \mathrm{kpc}$ in to $500 \mathrm{pc}$, typical of observations of dwarf and low surface brightness galaxy central densities, using the slope shown.

SIDM $\sigma_{\mathrm{DM}}=10^{-23} \mathrm{~cm}^{2} \mathrm{GeV}^{-1}$ halos are in good agreement with these observations, while $\sigma_{\mathrm{DM}}=$ $10^{-24} \mathrm{~cm}^{2} \mathrm{GeV}^{-1}$ produces inner densities that are a few times higher, but still marginally consistent with observations. In addition, $\rho_{c}$ in SIDM models show little trend with halo mass, in agreement with observations, because the core density is set by collisional physics.

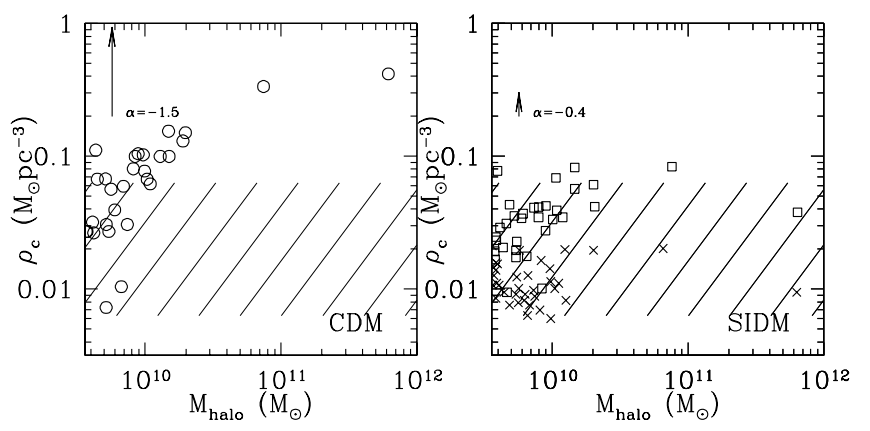

Figure 2: $\rho_{c}$ vs. $M_{\text {halo }}$ for CDM (left panel) and SIDM (right panel). For SIDM, crosses show $\sigma_{\mathrm{DM}}=10^{-23} \mathrm{~cm}^{2} \mathrm{GeV}^{-1}$, while open squares show $\sigma_{\mathrm{DM}}=10^{-24} \mathrm{~cm}^{2} \mathrm{GeV}^{-1}$. Only halos with 1000 or more particles are shown. The hatched region indicates the range of observed $\rho_{c}$ compiled by Firmani et al. (2000a). Arrow in upper left indicates how much each value of $\rho_{c}$ would increase if measured at $500 \mathrm{pc}$ (instead of $1 h^{-1} \mathrm{kpc}$ ), typical of observations, assuming a profile with the slope shown.

Conversely, the more massive halos in CDM have central densities that are too high by at least an order of magnitude already at $1 h^{-1} \mathrm{kpc}$, and because of their cuspy profile the disagreement would be much worse at smaller radii, as indicated by the arrow in the upper left. Moreover, CDM halos have central densities that increase with mass, in conflict with observations.

As such, SIDM halos appear to agree better with observations. Table 1 lists the median central density of halos with more than 1000 particles in our various models. This shows that our simulations reproduce the observed central halo densities for $\sigma_{\mathrm{DM}} \approx 10^{-23} \mathrm{~cm}^{2} \mathrm{GeV}^{-1}$.

\subsection{Inner Profile Slopes}

We estimate the inner halo profile slope $\alpha$ as the slope between the innermost resolved radii, $r=1 \rightarrow$ $1.5 h^{-1} \mathrm{kpc}$. Figure 3 shows a histogram of this slope for the collisionless (solid line), $10^{-24} \mathrm{~cm}^{2} \mathrm{GeV}^{-1}$ (dashed), and $10^{-23} \mathrm{~cm}^{2} \mathrm{GeV}^{-1}$ (dot-dashed) cases, for all halos that have more than 1000 particles. The number of such halos in each simulation is indicated in the legend. The qualitative impression from Figure 1 that SIDM produces shallower inner profiles is quantified in Figure 3. The median values of $\alpha$ are indicated by the arrows from the upper $x$-axis, and are listed in Table 1 .

CDM produces halos that have cuspy cores, with $\alpha_{\text {med }} \approx$ -1.5 . This is consistent with the work of Moore et al. among others. 25 of the $28 \mathrm{CDM}$ halos have $\alpha<-1$, indicating that cuspy cores are a common feature of CDM models. Conversely, the inner slopes in SIDM models are significantly shallower. For $\sigma_{\mathrm{DM}}=10^{-24} \mathrm{~cm}^{2} \mathrm{GeV}^{-1}$, $\alpha_{\text {med }} \approx-0.9$, while for $\sigma_{\mathrm{DM}}=10^{-23} \mathrm{~cm}^{2} \mathrm{GeV}^{-1}, \alpha_{\text {med }} \approx$ -0.4 , with no halos having $\alpha<-1$. This latter case has a median $\alpha$ close to the value preliminarily suggested by $\mathrm{H} \alpha$ observations of low surface brightness galaxies (Dalcanton $\&$ Bernstein 2000), though a definitive value awaits a more thorough analysis of observational biases.

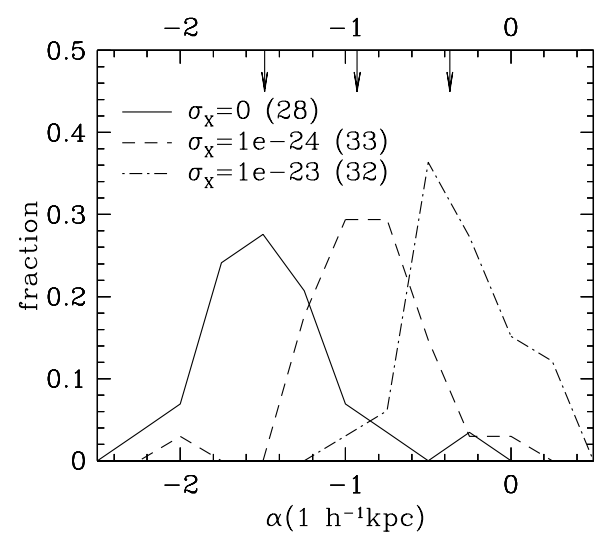

Figure 3: Histogram of inner slopes $\alpha$ for collisionless (solid), $10^{-24} \mathrm{~cm}^{2} \mathrm{GeV}^{-1}$ (dashed), and $10^{-23} \mathrm{~cm}^{2} \mathrm{GeV}^{-1}$ (dotdashed). Only halos with $M>3.6 \times 10^{9} M_{\odot}$ (> 1000 particles) are included; the number of such halos is indicated in parantheses in the legend. The median values of $\alpha$ are indicated by the arrows from the top edge of the plot, and are listed in Table 1.

The scatter in $\alpha$ is mostly real. There is some scatter due to discreteness effects in measuring the inner slope as we do. While we could obtain an inner slope from fitting a general halo shape (Hernquist 1990; Klypin 2000), with 5 free parameters the inner slope would be poorly constrained by 20 correlated data points, thus we choose our simpler definition. Further scatter arises from recently merged halos that temporarily have shallower profiles until relaxed. However, neither of these effects is very signifi- 
cant for the large mass halos plotted in Figure 3. Still, we choose to quote the median $\alpha$ rather than the mean, in order to quantify "typical" halos in these models and reduce sensitivity to outliers, although the mean is similar. We note that a significant scatter in inner slopes is also seen in the observations (e.g. de Blok, McGaugh \& van der Hulst 1996; Dalcanton \& Bernstein 2000).

Our CDM profiles are, at face value, in better agreement with the analytic profile of Moore et al. (1999), with an asymptotic slope of $\alpha=-1.5$, rather than an NFW profile having $\alpha(r \rightarrow 0)=-1$. However, profile fitting is a tricky business (as discussed in Klypin 2000). By reducing the scale radius in the NFW profile (i.e. increasing the concentration), one can push the transition to a slope of $\alpha=-1$ to a radius smaller than $1 h^{-1} \mathrm{kpc}$ where we cannot resolve the profiles (the "cusp-core degeneracy"; see van den Bosch \& Swaters 2000). Thus we suspect that our CDM profiles can also be adequately fit by an NFW profile having a large concentration parameter. As such, we do not argue for or against either profile form. Our simulations can only predict the slope at $r \approx 1 h^{-1} \mathrm{kpc}$, and that is what should be compared to observations.

SIDM appears to be in better agreement with observations of the inner slopes of dark halo profiles than CDM. At face value, $\sigma_{\mathrm{DM}}=10^{-23} \mathrm{~cm}^{2} \mathrm{GeV}^{-1}$ is preferred, but given uncertainties in observations and simulation techniques, $\sigma_{\mathrm{DM}}=10^{-24} \mathrm{~cm}^{2} \mathrm{GeV}^{-1}$ is probably also consistent. A similar value of $\sigma_{\mathrm{DM}}$ also reproduces the observed central density of galaxies. Such a coincidence is not expected a priori, and may represent a significant success of the SIDM scenario.

\subsection{Mass Dependence of Inner Slope}

Figure 4 shows a plot of inner halo slope $\alpha$ vs. halo mass $M_{\text {halo }}$ for all halos in our CDM (left panel) and SIDM (right panel, $\sigma_{\mathrm{DM}}=10^{-23} \mathrm{~cm}^{2} \mathrm{GeV}^{-1}$ ) simulations. The curve shows the running median value of $\alpha$ in bins of $\Delta(\log M)=0.5$.

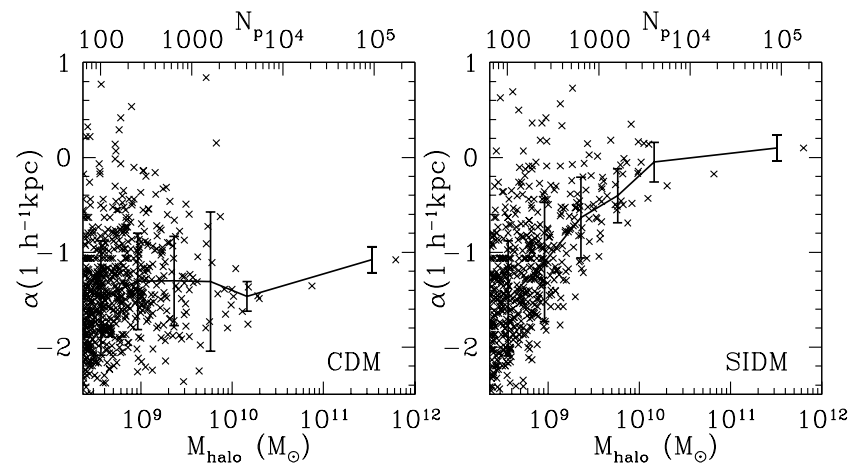

Figure 4: $\alpha$ vs. $M_{\text {halo }}$ for CDM (left panel) and SIDM $\left(\sigma_{\mathrm{DM}}=10^{-23} \mathrm{~cm}^{2} \mathrm{GeV}^{-1}\right.$; right panel). All halos with 64 or more particles are shown. The line shows a running median of the $\alpha$ distribution, with a variance computed from all halos within each mass bin.

The CDM case shows almost no trend with mass, with the median slope always around $-1.2 \sim-1.5$. The largest halo does have a slightly shallower slope, consistent with the trend seen in very high resolution CDM simulations of individual halos (Jing \& Suto 2000). The scatter increases to low mass due to discreteness and merging effects de- scribed in $\$ 3.3$.

The SIDM case shows dramatically different behavior, suggesting at face value that smaller halos have steeper inner profiles. However, caution must be used in interpreting this result. First, smaller halos have smaller cores, meaning that a slope measured at a fixed radius (not scaled to the halo core size) will result in a steeper slope. For CDM this effect is less significant, since the slope remains similar from the outer to the inner halo. Second, the small numbers of particles in the low-mass halos makes the Monte Carlo technique less effective in modeling collisions, thereby making SIDM appear more like CDM; we investigate this issue further in \$4. Thus we make no claim regarding a trend of $\alpha$ with $M_{\text {halo. }}$.

\subsection{Mass Concentration Parameter}

As seen in Figure 1, SIDM appears to have the desired effect of reducing the concentration of dark matter halos. In this section we quantify this effect using a concentration parameter, which we define differently than previous authors in order to facilitate a more direct comparison with observations.

The canonical definition of a concentration parameter is given by NFW as the ratio between the virial radius $r_{200}$ (taken to be the radius at which the halo density is $200 \times$ the cosmic mean) and the scale radius of the halo $r_{s}$ in the NFW profile. This concentration parameter, however, is difficult to compute unambiguously in the case of non-isolated halos, and difficult to compare directly to observations that seldom extend out to $r_{200}$. Furthermore, $r_{s}$ is only defined within the context of the specific NFW model, and profile fits are typically degenerate between $r_{s}$ and concentration (Klypin 2000). Colín et al. (2000) circumvent some of these issues by defining the concentration as the ratio of the minimum of $r_{200}$ and the halo radius to the radius that encloses $20 \%$ of the halo mass. However, this inner radius is dependent on knowing the total halo mass, something which is difficult to determine observationally.

Instead, we choose to define a mass concentration $p a-$ rameter $c_{M}$, based on enclosed mass rather than radii, and restrict the scales in our definition to those where observations are available, typically $r \lesssim 20 h^{-1} \mathrm{kpc}$. We define

$$
c_{M}=27 \frac{M\left(<r_{\text {in }}\right)}{M\left(<r_{\text {out }}\right)}
$$

with

$$
r_{\text {in }}=\frac{1}{3} r_{\text {out }}=8.5 \mathrm{kpc} \frac{v_{\text {circ }}}{220 \mathrm{~km} \mathrm{~s}^{-1}},
$$

where $v_{\text {circ }}$ is the circular velocity of the halo. The choice of $r_{\text {in }}$ is arbitrary; here we base it on the Milky Way, as it is convenient and results in observationally accessible scales. The scaling with $v_{\text {circ }}$ is that expected for self-similar halos following the Tully-Fisher relation. The normalization factor of 27 results in a uniform density distribution having a mass concentration of unity. A flat rotation curve between $r_{\text {in }}$ and $r_{\text {out }}$ implies $M(r) \propto r$, resulting in $c_{M}=9$. In our simulations, we take $v_{\text {circ }}$ to be the maximum circular velocity of the halo as output by SKID. 


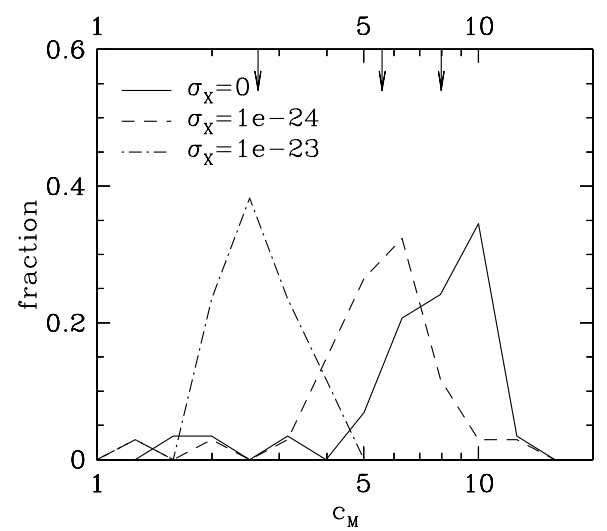

Figure 5: Histogram of mass concentrations $c_{M}$ for collisionless (solid), $10^{-24} \mathrm{~cm}^{2} \mathrm{GeV}^{-1}$ (dashed), and $10^{-23} \mathrm{~cm}^{2} \mathrm{GeV}^{-1}$ (dot-dashed). Only halos with $M>3.6 \times 10^{9} M_{\odot}$ (> 1000 particles) are included. The median values of $c_{M}$ are indicated by the arrows from the top edge of the plot, and are listed in Table 1.

Figure 5 shows a histogram of $c_{M}$ for all halos with more than 1000 particles. As expected, there is a clear trend for CDM to have more concentrated halos than SIDM, with the amount of concentration decreasing with increasing $\sigma_{\mathrm{DM}}$. Note that the difference between SIDM with $10^{-24} \mathrm{~cm}^{2} \mathrm{GeV}^{-1}$ and $10^{-23} \mathrm{~cm}^{2} \mathrm{GeV}^{-1}$ is exaggerated relative to the difference between the inner slopes of those models (cf. Figure 3). This is because the concentration is increased in $10^{-24} \mathrm{~cm}^{2} \mathrm{GeV}^{-1}$ relative to $10^{-23} \mathrm{~cm}^{2} \mathrm{GeV}^{-1}$ both due to the increased inner slope, as well as the reduced core radius. We also examined the mass dependence of $c_{M}$ and found no obvious trend, but our range of masses is small.

For comparative purposes, our mass concentration parameter $c_{M}$ may be analytically related to the NFW concentration parameter $c_{\mathrm{NFW}}$. From NFW,

$$
\frac{v_{\mathrm{c}}^{2}(x)}{v_{2 \rho \rho}^{2}}=\frac{1}{x} \frac{\ln \left(1+c_{\mathrm{NFW}} x\right)-c_{\mathrm{NFW}} x /\left(1+c_{\mathrm{NFW}} x\right)}{\ln \left(1+c_{\mathrm{NFW}}\right)-c_{\mathrm{NFW}} /\left(1+c_{\mathrm{NFW}}\right)},
$$

where $x=r / r_{200}, v_{\mathrm{c}}(x)$ is the circular velocity at $x$, and $v_{200}$ is the circular velocity at $r_{200}$. Our $v_{\text {circ }}$ is taken to be the maximum halo circular velocity, which may obtained by maximizing equation 8 ; this occurs at $x_{\max } \approx 2 / c_{\mathrm{NFW}}$ (though we compute it exactly for the results shown below).

Let $\hat{v}_{\text {circ }} \equiv v_{\mathrm{c}}\left(x_{\max }\right) / v_{200}$. In appropriate units, $v_{200}=$ $r_{200}$ (see NFW, equation A2). Thus

$$
x_{\text {in }} \equiv r_{\text {in }} / r_{200}=\frac{8.5}{220} \hat{v}_{\text {circ }}
$$

implying $x_{\mathrm{in}}$ and $x_{\mathrm{out}}=3 x_{\mathrm{in}}$ are solely functions of $c_{\mathrm{NFW}}$ (note that this arises because we defined $r_{\text {in }} \propto v_{\text {circ }}$ ). Using $M(<r) \propto r v^{2}(r)$.

$$
c_{M}=9 \frac{v_{c}^{2}\left(x_{\mathrm{in}}\right)}{v_{c}^{2}\left(x_{\mathrm{out}}\right)},
$$

which is purely a function of $c_{\mathrm{NFW}}$. The resulting relationship is shown in Figure 6.

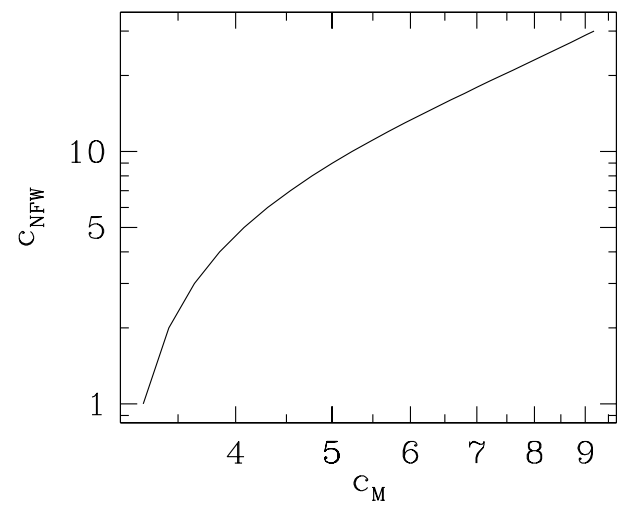

Figure 6: Mass concentration parameter $c_{M}$, defined in equation 6, vs. NFW concentration parameter $c_{\mathrm{NFW}}$.

Figure 6 shows that $c_{M} \approx 8$, typical of halos in our $\mathrm{CDM}$ model, corresponds to $c_{\mathrm{NFW}} \approx 23$. This value is in agreement with expectations for dwarf galaxies in a $\Lambda \mathrm{CDM}$ model. Conversely, $c_{M} \approx 5.6$, which is the median value for SIDM with $10^{-24} \mathrm{~cm}^{2} \mathrm{GeV}^{-1}$, corresponds to $c_{\mathrm{NFW}} \approx 11$. Note that the minimum value of $c_{M}$ for an NFW halo is 3. Thus SIDM with $10^{-23} \mathrm{~cm}^{2} \mathrm{GeV}^{-1}$, with $c_{M \text {,med }} \sim 2.6$, produces halos that typically cannot be described properly by $c_{\mathrm{NFW}}$. This is because these halos have $\alpha>-1$ typically, so NFW profiles with $\alpha(r \rightarrow 0)=-1$ are a poor fit. This further illustrates why $c_{\mathrm{NFW}}$ is a poor way to describe halos in general.

The largest halo in our simulations has a mass comparable to the Milky Way's, $\approx 6 \times 10^{11} M_{\odot}$. The concentrations of this halo are 6.7, 4.4 and 2.3 in CDM, $\sigma_{\mathrm{DM}}=10^{-24} \mathrm{~cm}^{2} \mathrm{GeV}^{-1}$ and $\sigma_{\mathrm{DM}}=10^{-23} \mathrm{~cm}^{2} \mathrm{GeV}^{-1}$, respectively. $c_{M}$ of the Milky Way halo is somewhat uncertain, because of the uncertainty in the rotation curve outside the solar circle $\left(R_{\odot}\right)$ and the effect of baryonic mass within $R_{\odot}$, but we make a rough estimate here. If the rotation curve is flat, $c_{M}=9$ as stated before. There are suggestions that the rotation curve rises somewhat beyond the solar circle (though this is uncertain; see Olling \& Merrifield 2000), in which case $c_{M}$ is reduced; let us take $c_{M}=8$ as a working estimate. The rotation curve measures the total mass, so we must correct for the baryons to compare with our simulated $c_{M}$. If we take the fraction of baryonic mass to be $50 \%$ inside $R_{\odot}$ and $20 \%$ inside $3 R_{\odot}$, then $c_{M}$ reduces to 5 . In addition, baryons adiabatically compress the dark matter as they dissipate, so we must correct the Milky Way $c_{M}$ further downwards to compare to our dissipationless halos. From the analysis of Avila-Reese, Firmani \& Hernandez (1998), this reduction factor is $\sim 1.5-2$, resulting in the Milky Way halo having $c_{M} \sim 3$. Thus after reasonable corrections, the Milky Way mass concentration appears to be in better agreement with SIDM than CDM. The rapid rotation of bars also suggests a lower concentration for the Milky Waysized galaxies than that predicted by CDM de Battista \& Sellwood 1998).

A more direct comparison with simulations may be obtained from rotation curves of dark matter-dominated, low surface brightness galaxies, where baryonic corrections are smaller. We expect that this mass concentration measure $c_{M}$ will be relatively straightforward to compute from such 
rotation curves (e.g. Dalcanton \& Bernstein 2000), so we look forward to comparisons. $c_{M}$ has the advantage that it is independent of halo fitting parameters, as the enclosed mass can be obtained directly from the observed circular velocity with modest assumptions. In this sense, it is a more robust comparison than the inner slope and the NFW concentration parameter, which are degenerate and sensitive to scales outside those typically observed van den B osch \& Swaters 2000), and the central density, which depends on an uncertain contribution from baryons.

\subsection{Phase Space Densities}

A recently popularized measure of the concentration of dark matter halos is the central phase space density. Dalcanton \& Hogan (2000) find that observed phase space densities $Q \equiv \rho / \sigma^{3}$ scale as $Q \propto \sigma^{-3}-\sigma^{-4}$, where $\sigma$ is the velocity dispersion, from dwarf spheroidals up to clusters of galaxies. Observations compiled by Sellwood (2000) suggest a similar relation, albeit with a large scatter, and he uses them to argue against any form of collisionless dark matter (though see Madsen 2000).

Figure 7 shows the phase space density $Q$ of dark matter within $r_{\text {in }}$ as a function of $\sigma$, for halos with $\sigma>30 \mathrm{~km} \mathrm{~s}^{-1}$. We calculate $\sigma$ as the velocity dispersion around the group center of mass velocity, within $r_{\text {in }}$ (cf. equation 7). Open circles show CDM halos, crosses indicate $\operatorname{SIDM}\left(\sigma_{\mathrm{DM}}=\right.$ $\left.10^{-23} \mathrm{~cm}^{2} \mathrm{GeV}^{-1}\right)$ halos. The dashed and dotted lines show $Q \propto \sigma^{-3}$ and $Q \propto \sigma^{-4}$, respectively, that bracket the observations, reproduced from Dalcanton \& Hogan (2000).

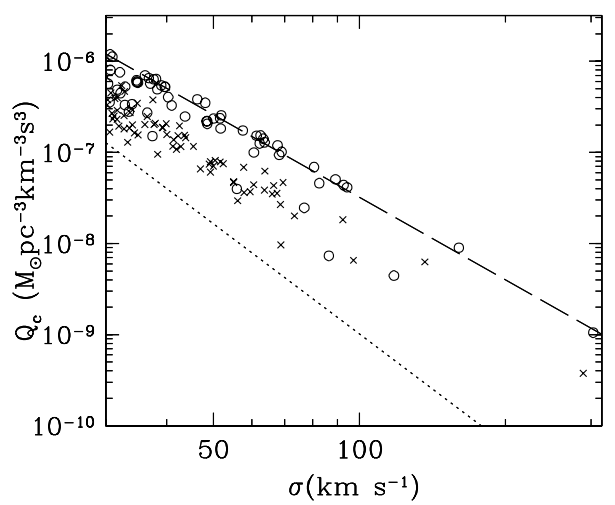

Figure 7: Phase space density $Q$ vs. velocity dispersion $\sigma$ for CDM (open circles), and SIDM with $\sigma_{\mathrm{DM}}=10^{-23} \mathrm{~cm}^{2} \mathrm{GeV}^{-1}$ (crosses). Dashed and dotted lines bracket observations, showing scalings of $Q \propto \sigma^{-3}$ and $Q \propto \sigma^{-4}$, respectively.

SIDM generally shows lower phase space densities than CDM. SIDM is in somewhat better agreement with observations, falling in the middle of the observed range. Measuring $Q$ in galaxies is a difficult task, because the stellar velocity dispersion is not necessarily that of the dark matter. Furthermore, in rotationally supported galaxies the dubious assumption of an isothermal spherical halo is used to relate circular velocity to dispersion. Thus $Q$ is perhaps not among the most useful observational discriminants between CDM and SIDM.

An interesting remark from Figure 7 is that the scaling of $Q(\sigma)$ is roughly the same in both models, roughly $Q \propto \sigma^{-3}$. Dalcanton \& Hogan (2000) argue that such a scaling results from the dynamical assembly of halos, and is not expected based on simple phase packing arguments. This further motivates simulations of SIDM that include the cosmological growth of halos via dynamical processes of merging and accretion.

\subsection{Ellipticities}

SIDM produces halos that are more spherical than CDM, because of the isotropic nature of the collisions (Spergel \& Steinhardt). This is a generic feature of SIDM with any significant cross section, since in the inner portions of halos where collisions are frequent, the velocity ellipsoid is quickly isotropized. Thus the shapes of dark matter halos provide an important observational discriminant between CDM and SIDM.

We compute axis ratios of our halos using the prescription outlined in Dubinski \& Carlberg (1991). They define a tensor

$$
M_{i j}=\sum \frac{x_{i} x_{j}}{a^{2}}, \quad \text { with } a \equiv\left(x_{1}^{2}+\frac{x_{2}^{2}}{q^{2}}+\frac{x_{3}^{2}}{s^{2}}\right)^{\frac{1}{2}},
$$

where $a$ is the elliptical radius, and $s \leq q \leq 1$ are the axis ratios, and the sum is over all particles with distances $\approx\left(x_{1}, x_{2}, x_{3}\right)$ from the halo center along the axes of the ellipsoid. Then,

$$
q=\left(\frac{M_{y y}}{M_{x x}}\right)^{\frac{1}{2}} \text { and } s=\left(\frac{M_{z z}}{M_{x x}}\right)^{\frac{1}{2}},
$$

where $M_{x x} \geq M_{y y} \geq M_{z z}$ are the eigenvalues of $M . q$ represents the axisymmetry of the halo, while $s$ measures the halo flattening. Since $a$ depends on $q$ and $s$, the calculation of $M_{i j}$ must be iterated until convergence, which we take to be better than 0.01 in $q$ and $s$. This scheme weights particles roughly equally regardless of distance from center, unlike a moment of inertia tensor which weights the outskirts heavily, and thus better represents the ellipticity of the density distribution, as shown in Dubinski \& Carlberg (1991).
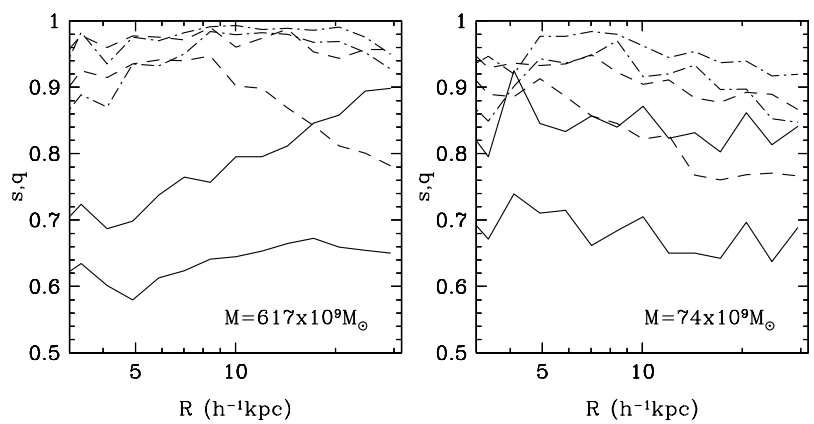

Figure 8: Axisymmetry $q$ and flattening $s$ vs. $r$ for a $6 \times 10^{11} M_{\odot}$ halo (left panel) and a $7 \times 10^{10} M_{\odot}$ halo (right panel). Solid lines are CDM, dashed lines are SIDM, $\sigma_{\mathrm{DM}}=10^{-24} \mathrm{~cm}^{2} \mathrm{GeV}^{-1}$, and dot-dashed lines are SIDM, $\sigma_{\mathrm{DM}}=10^{-23} \mathrm{~cm}^{2} \mathrm{GeV}^{-1}$. Lower of two curves is $s$.

Figure 8 shows axis ratios as a function of radius in our two most massive halos, having masses $\approx 6 \times$ $10^{11} M_{\odot}$ (left panel) and $\approx 7 \times 10^{10} M_{\odot}$ (right panel) Solid line is the CDM halo, dashed line is SIDM with $\sigma_{\mathrm{DM}}=10^{-24} \mathrm{~cm}^{2} \mathrm{GeV}^{-1}$, and dot-dashed line is SIDM 
with $\sigma_{\mathrm{DM}}=10^{-23} \mathrm{~cm}^{2} \mathrm{GeV}^{-1} \cdot q$ is the upper of two curves for a given model.

CDM halos are fairly triaxial, while SIDM produces halos that are much closer to spherical. The effect is dependent on radius, as in the outer regions SIDM and CDM become more similar, since the effect of self-interactions is confined to the inner parts of halos. Still, even at $30 h^{-1} \mathrm{kpc}$ there are significant differences between SIDM and CDM. While not stated, this trend with radius is also evident from Figure 1 of Yoshida et al. (2000b).

Figure 9 shows histograms of axis ratios $q$ and $s$ at $2 h^{-1} \mathrm{kpc}$ (top panels) and $10 h^{-1} \mathrm{kpc}$ (bottom panels) for all halos with more than 1000 particles. The median value for each model is indicated by the corresponding tick mark on the top axis. The difference between CDM and SIDM is more pronounced at small radii, where CDM produces significantly triaxial halos while SIDM halos remain spherical. At large radii there is a much milder trend to more spherical halos with increasing $\sigma_{\mathrm{DM}} . s$ also shows more differences than $q$.

Figure 9 shows that while CDM produces halos are typically more spherical, there is still significant non-sphericity in many SIDM halos. In particular, there is a tail in the distributions of both CDM and SIDM to smaller axis ratios. This may be due to asymmetric infall that temporarily distorts the shape of the density in some halos, particularly smaller ones. This also may just be an artifact of finite number of collisions in smaller halos. Note that the two largest halos shown in Figure 8 show greater differences at $10 h^{-1} \mathrm{kpc}$ than suggested by the statistics in Figure 9.
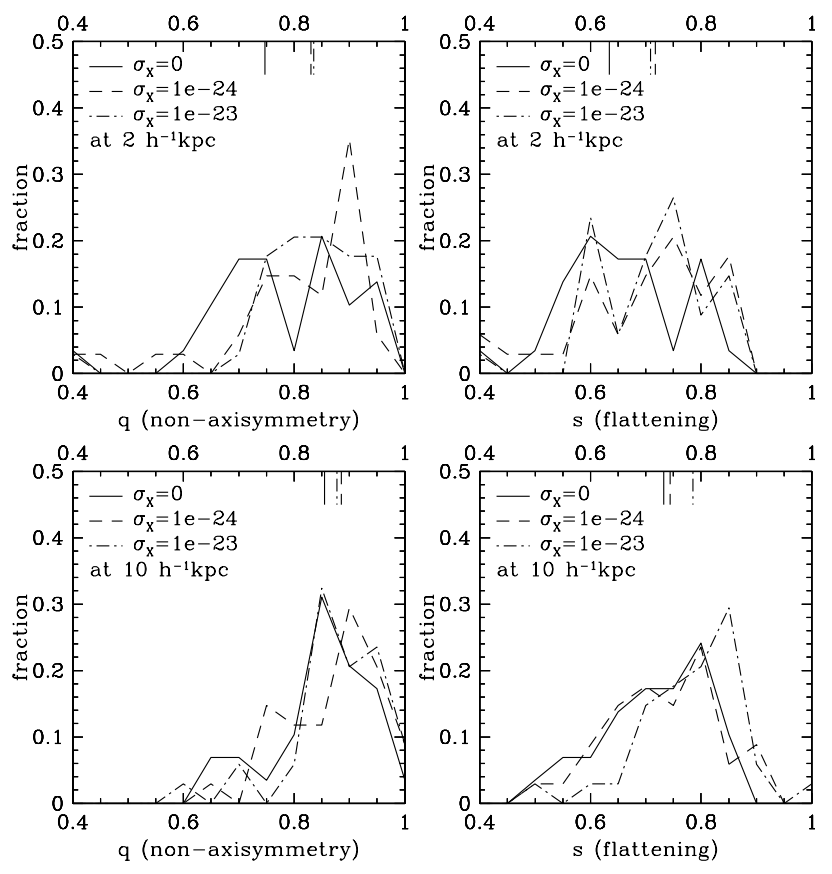

Figure 9: Histogram of axis ratios $q$ (left panels) and $s$ (right panels), at $2 h^{-1} \mathrm{kpc}$ (upper panels) and $10 h^{-1} \mathrm{kpc}$ (lower panels). Only halos with more than 1000 particles are included. Tick marks at the upper axis show median values.

A comparison with observations of halo shapes is as yet inconclusive. In the inner portions of dark halos the shape of the potential is likely to be dominated by baryons, so a comparison to these simulations is not straightforward. Further out, perhaps the most direct observations of axisymmetry are those for galaxies with $\mathrm{HI}$ rings such as IC 2006, which suggests a very axisymmetric halo, $q \approx 0.93 \pm 0.08$ at $\approx 13 \mathrm{kpc}$ (Franx, van Gorkom \& de Zeeuw 1994). Other observations (see Sackett 1999) are more dependent on observational and theoretical uncertainties such as viewing angle and potential modeling, but persistently suggest $q \gtrsim 0.8$ at $\sim 15-20 \mathrm{kpc}$. Both CDM and SIDM halos are consistent with these observations. Lensing maps of galaxies and clusters offer the best hope for mapping the mass potential in the inner halos, which should place strong constraints on SIDM.

Conversely, observations of $s$ from polar ring galaxies (e.g. Sackett et al. 1994) and X-ray isophotes (e.g. Buote \& Canizares 1998) suggest a substantial amount of flattening, $s \approx 0.5 \pm 0.2$ at $r \sim 15 \mathrm{kpc}$ in the density distribution. Such a flattening, if confirmed, may prove troublesome for SIDM. The baryonic component would provide a flattened contribution, but is not expected to be significant at those radii. It is not immediately evident how these discrepancies may be resolved, but we note that the problem is almost as severe for CDM as SIDM in our simulations. It is worth mentioning that our small simulation volume results in significantly reduced tidal distortion of large halos, so our simulations may not accurately represent the ellipticities of the outer portions of halos.

\section{THE MONTE CARLO RESOLUTION LIMIT}

Our spatial resolution and mass resolution are wellunderstood. However, another resolution issue arises due to the Monte Carlo modeling of self-interactions. A Monte Carlo method must be sufficiently well sampled, resulting in a separate criterion for the number of particles in a halo to be well-represented by our simulation technique. In this section, we determine this criterion using our suite of lower resolution simulations with $64^{3}$ particles described in $\$ 2$.

Since we are most concerned with the inner parts of halos, we focus on the inner slope as a function of mass as the best measure for examining this Monte Carlo resolution limit. Figure 10 shows a plot similar to right panel of Figure 4, except for the $64^{3}$ simulation of the $\sigma_{\mathrm{DM}}=10^{-23} \mathrm{~cm}^{2} \mathrm{GeV}^{-1}$ SIDM model. The top axis shows the number of particles in these halos. Here we compute $\alpha$ at $2 h^{-1} \mathrm{kpc}$ since that is the spatial resolution of our $64^{3}$ runs. The dashed line is the running median $\alpha$ from the $128^{3}$ simulation, computed at $2 h^{-1} \mathrm{kpc}$.

Figure 10 shows that for halos with $\gtrsim 1000$ particles in the $64^{3}$ run, the median value of $\alpha$ is within $1 \sigma$ of that of the $128^{3}$ run, though consistently lower. By 300 particles, the value of $\alpha$ is significantly lower in the $64^{3}$ run. The reason it is lower is because with few particles, the Monte Carlo procedure results in too few interactions to make the profile depart significantly from the collisionless CDM case. Thus to lower masses, SIDM looks increasingly like CDM when modeled using this technique. 


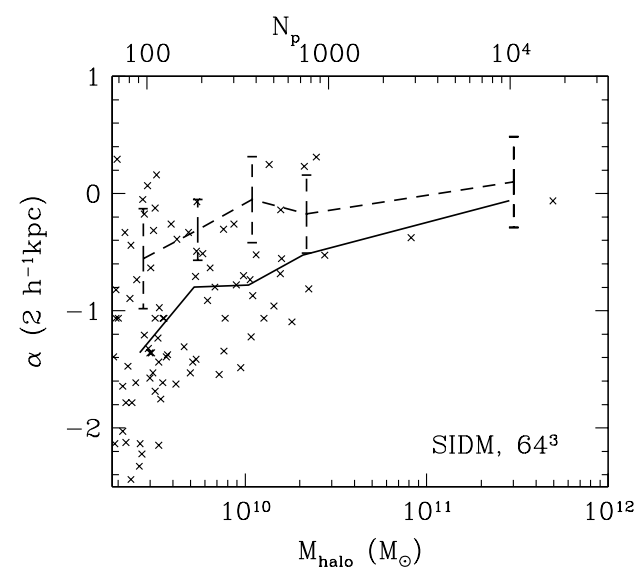

Figure 10: $\alpha$ vs. $M_{\text {halo }}$ for SIDM with $\sigma_{\mathrm{DM}}=$ $10^{-23} \mathrm{~cm}^{2} \mathrm{GeV}^{-1}$, for our $64^{3}$ run. $\alpha$ here is measured at $2 h^{-1} \mathrm{kpc}$, the resolution of our $64^{3}$ runs. Solid line shows a running median of the $\alpha$ distribution. Dashed line with error bars shows a similar curve from $128^{3}$ run. Deviations between two at a level $>1 \sigma$ occur for halos having somewhere between 300 and 1000 particles.

Note that this limit is specific to our simulation parameters, redshift, and $\sigma_{\mathrm{DM}}$, and is not a general statement about the Monte Carlo $N$-body technique. The limit becomes higher as $\sigma_{\mathrm{DM}}$ is lowered, since collisions become less frequent, but even for $\sigma_{\mathrm{DM}}=10^{-24} \mathrm{~cm}^{2} \mathrm{GeV}^{-1}$, $1000+$ particle halos also appear convergent. We therefore take our Monte Carlo resolution limit to be $\gtrsim 1000$ particles.

We list the values of $\alpha_{\text {med }}$ and $c_{M \text {,med }}$ for the $64^{3}$ runs in Table 1 for comparison with the $128^{3}$ results, where the median values here are computed for all halos with more that 500 particles in these smaller runs (roughly 10 in each simulation). Note that $\alpha_{\text {med }}$ is computed at $2 h^{-1} \mathrm{kpc}$ instead of $1 h^{-1} \mathrm{kpc}$, partially explaining the steeper slopes even at the highest masses. In general, the trends indicated by the $128^{3}$ runs are reproduced at this lower resolution, suggesting that discrete particle effects do not significantly affect our conclusions. We have also examined the $128^{3}$ statistics presented previously using a limit of 300 particles instead of 1000, and our overall conclusions remain the same.

\section{SUBHALO POPULATION}

Self-interacting dark matter is predicted to significantly lower the population of subhalos orbiting around large halos, thereby bringing simulation predictions into better agreement with observations of the Local Group dwarf population. There are two reasons why SIDM has this effect: (1) The lowered central concentration and larger core radius makes small halos more susceptible to tidal disruption, and (2) Dark matter is ram-pressure stripped out of small galaxies as they move through the large central halo. In our $128^{3}$ simulations, we have one halo that is roughly Milky Way sized, having $M \approx 6.7 \times 10^{11} M_{\odot}$. In this section we examine the subhalo population around this large halo.

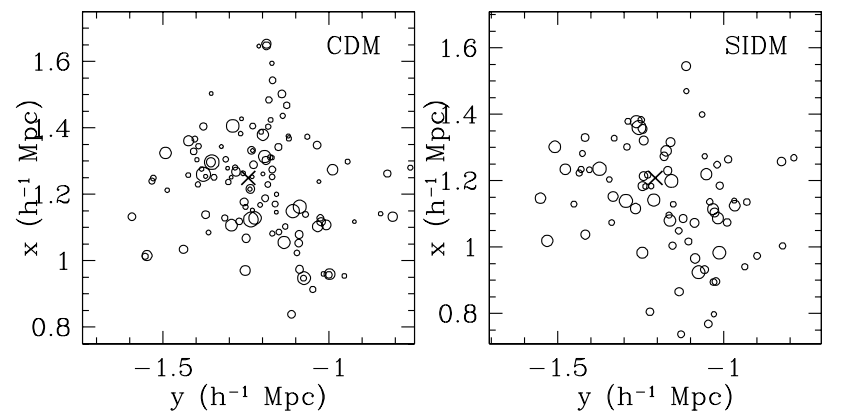

Figure 11: Subhalo positions within $500 h^{-1} \mathrm{kpc}$ the largest object in our volume, in CDM (left panel) and SIDM with $\sigma_{\mathrm{DM}}=10^{-23} \mathrm{~cm}^{2} \mathrm{GeV}^{-1}$ (right panel). Central cross is the position of the large halo.

Figure 11 shows a projected plot of halos within $500 h^{-1} \mathrm{kpc}$ of the largest halo in our volume, indicated by the central cross. Circle sizes are scaled as $\log \left(M_{\text {halo }}\right)$, with the smallest circles representing halos with $M \approx$ $5 \times 10^{8} M_{\odot}$. Left panel shows CDM, right panel shows SIDM with $\sigma_{\mathrm{DM}}=10^{-23} \mathrm{~cm}^{2} \mathrm{GeV}^{-1}$. The positions of subhalos are different due to the accumulated differences of chaotic orbits within a highly nonlinear potential well. Thus a halo-by-halo comparison for these small halos is not possible. A careful examination reveals that SIDM has fewer subhalos than the CDM distribution, especially the smallest ones.

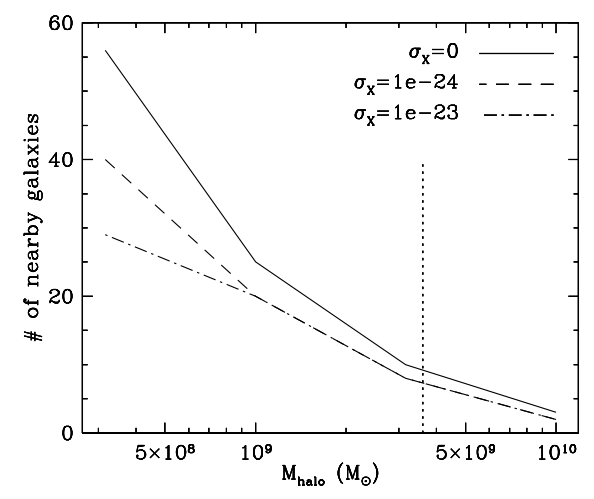

Figure 12: Number of halos within $500 h^{-1} \mathrm{kpc}$ of the largest halo, histogrammed by mass, for collisionless (solid), $10^{-24} \mathrm{~cm}^{2} \mathrm{GeV}^{-1}$ (dashed), and $10^{-23} \mathrm{~cm}^{2} \mathrm{GeV}^{-1}$ (dotdashed). Vertical dotted line indicates our 1000-particle Monte Carlo resolution limit.

Figure 12 quantifies this effect, showing the mass function of halos within $500 \mathrm{~h}^{-1} \mathrm{kpc}$ of our largest halo, for our three $128^{3}$ simulations. There is a clear trend that SIDM suppresses the subhalo population at the smallest masses. For $10^{8.5}<M<10^{9} M_{\odot}$, CDM has 56 neighboring halos, $\sigma_{\mathrm{DM}}=10^{-24} \mathrm{~cm}^{2} \mathrm{GeV}^{-1}$ has 40 , and $\sigma_{\mathrm{DM}}=10^{-23} \mathrm{~cm}^{2} \mathrm{GeV}^{-1}$ has 29 .

While SIDM does reduce the population of smallest halos, the reduction is not nearly by the order of magnitude required to obtain agreement with Local Group dwarf galaxy counts (Moore et al. 1999a). However, the effect of subhalo suppression in these SIDM simulations should 
be regarded as a lower limit to the true strength of the effect. The reason is that almost all these subhalos are well below our Monte Carlo resolution limit (dotted line in Figure 12), therefore their concentrations are approaching those in CDM models. Thus tidal disruption of these halos is not much stronger in SIDM as in CDM, and is increasingly similar to lower masses. Furthermore, rampressure stripping is reduced in effectiveness for the same reason that the Monte Carlo technique is less effective in these small halos. Hence the numbers of small SIDM halos are not as significantly suppressed relative to CDM as they should be.

We conclude that the simulations considered here suggest a weak trend in reducing the number of subhalos with increasing $\sigma_{\mathrm{DM}}$, but due to resolution effects we can make no robust quantitative estimates. What is required is to simulate a large halo with incredibly high resolution, having subhalos containing thousands of particles to properly model the effects of self-interactions. Such a simulation is unfortunately beyond the scope of our current computational resources. Alternatively, more sophisticated algorithms are necessary to model self-interactions in small halos moving through large ones, which is an avenue we are currently pursuing.

\section{COMPARISON WITH PREVIOUS WORK}

A number of authors have investigated SIDM using $N$ body simulations. The literature divides into two subsets: Those that model interactions in the fluid approximation, effectively employing a large cross section, and those that model self-interactions in the optically thin regime as suggested by Spergel \& Steinhardt. In both cases, there is disagreement over whether SIDM makes halos less or more concentrated than CDM.

Moore et al. (2000) and Yoshida et al. (2000a) simulate a galaxy cluster within a cosmological context, using a treecode with $\mathrm{SPH}$ to model interactions in the fluid approximation. Both studies resulted in halos that had isothermal profiles and were more centrally concentrated than CDM halos. This may be because the large effective cross section increases heat transfer efficiency, though as mentioned in the Introduction, for a sufficiently large cross section one expects heat transfer to be diminished. Yoshida et al. (2000a) suggested that intermediate cross sections would likely yield results that were intermediate between steep CDM profiles and steeper isothermal profiles, and thereby argued against SIDM. However, this is contradicted by Yoshida et al. (2000b), as well as the results presented here, confirming that the intermediate case results in halos that have long-lived shallow profiles.

In contrast, Bryan (private communication) uses a adaptive mesh hydrodynamics code to model self-interactions in a cosmological volume, and finds that even in the fluid limit SIDM produces sizeable, long-lived cores. It is not clear why different hydrodynamic codes give different results when they should be operating in the same regime. Perhaps the effective cross section is larger in the adaptive mesh code due to algorithmic differences, reducing heat transfer. Another possibility is that a numerical effect in SPH in which cold clumps moving through hot halos have their drag significantly overestimated (Tittley, Couchman \& Pearce 1999) makes objects rapidly sink into a dense, isothermal core. It is beyond the scope of this paper to resolve these issues. We simply note that the highly optically thick limit is not the relevant scenario to test the cross section range proposed by Spergel \& Steinhardt.

Burkert (2000) and Kochanek \& White (2000) simulated isolated halos with SIDM having a cross section closer to the range of Spergel \& Steinhardt. They begin with a fully formed cuspy galaxy halo and study the evolution after interactions are turned on. They both find that halos develop a shallow core for some length of time, and then undergo core collapse. Burkert and Kochanek \& White disagree on the timescales of core collapse; Burkert finds $t_{c} \sim 16 t_{\mathrm{dyn}}$ in agreement with estimates from two-body relaxation, while Kochanek \& White finds a much shorter collapse timescale of $t_{c} \sim 2 t_{\mathrm{dvn}}$ for the same dark matter cross section. Kochanek \& White explain this difference by arguing that Burkert's method underestimates collisions of slow moving particles. We note that our method does not suffer from this concern, as it is more like Kochanek \& White.

We suggest some possible reasons why the results of Kochanek \& White and (to a lesser extent) Burkert are at odds with ours. The first is that they begin with a cuspy Hernquist profile. This halo evolves rapidly initially (as seen in Figure 3 of Kochanek \& White, resulting in an artificially large amount of heat transfer. Second, they simulate an isolated halo, ignoring the accretion of dynamically hot material during the formation process that would keep the outer halo hot and delay core collape. We note that their dimensionless cross section $\hat{\sigma}_{D M}=M_{\text {halo }} \sigma_{\mathrm{DM}} / r_{s}^{2}$ converts to ours by a factor of $\sim 2 \times 10^{-23} \mathrm{~cm}^{2} \mathrm{GeV}^{-1}$ for $M_{\text {halo }} \approx 2 \times 10^{10} M_{\odot}$ and $r_{s} \approx 5 h^{-1} \mathrm{kpc}$ (cf. Figure 1). Kochanek \& White's simulation with $\hat{\sigma}_{D M}=1$ already produces halos that maintain cores over many dynamical times (cf. top panel of their Figure 2). We suggest that a somewhat smaller $\hat{\sigma}_{D M}$ might be consistent with observations as well as their limits on core collapse timescales, even without considering the effects of accretion and merging.

Kochanek \& White also point out, as we have, that the Monte Carlo $N$-body technique requires a large number of particles for accurate modeling, and show that $10^{5}$ particles is sufficient. We note that while most of our halos do not have that many particles, our largest (Milky Way sized) halo has roughly $2 \times 10^{5}$ particles, and its properties are consistent with those of smaller halos.

Yoshida et al. (2000b) have now performed a cosmological cluster simulation with a cross sections $\sigma_{\mathrm{DM}} \approx$ $2 \times 10^{-25}-2 \times 10^{-23} \mathrm{~cm}^{2} \mathrm{GeV}^{-1}$ using a Monte Carlo $N$-body method. They find shallower central slopes and less concentrated cores with SIDM. Our combined results span the range from dwarf galaxies to clusters, and are in broad agreement with each other if we focus purely on the numerical results. For instance, they find that $\sigma_{\mathrm{DM}} \approx 2 \times 10^{-23} \mathrm{~cm}^{2} \mathrm{GeV}^{-1}$ produces a cluster having a core size of $160 h^{-1} \mathrm{kpc}$. If we extrapolate our largest halo's core size (in our $\sigma_{\mathrm{DM}}=10^{-23} \mathrm{~cm}^{2} \mathrm{GeV}^{-1}$ run) to cluster scales using the expected $r_{\text {core }} \propto v_{\text {circ }}$, we predict a core size of $\sim 150 h^{-1} \mathrm{kpc}$ for their cluster. For $\sigma_{\mathrm{DM}} \approx$ $2 \times 10^{-24} \mathrm{~cm}^{2} \mathrm{GeV}^{-1}$, they get $100 h^{-1} \mathrm{kpc}$ core, while we would predict $\sim 80 h^{-1} \mathrm{kpc}$ for $\sigma_{\mathrm{DM}}=10^{-24} \mathrm{~cm}^{2} \mathrm{GeV}^{-1}$, again in good agreement. They then proceed to scale down from clusters to dwarf galaxies using a simplistic argument 
based on number of collisions, but their scaled result is contradicted by our direct simulations. We estimate that this may be because they use a CDM value for the scale radius and $c_{\mathrm{NFW}}$ of dwarfs, and compare them to SIDM values for their cluster. We find that $\sigma_{\mathrm{DM}}=10^{-24} \mathrm{~cm}^{2} \mathrm{GeV}^{-1}$ produces NFW scale radii that are double that of CDM (cf. Figure 6 and discussion); such a factor would go a long way towards alleviating the discrepancy. Taking this into account, we find the simulations of Yoshida et al. (2000b) to be broadly consistent with ours.

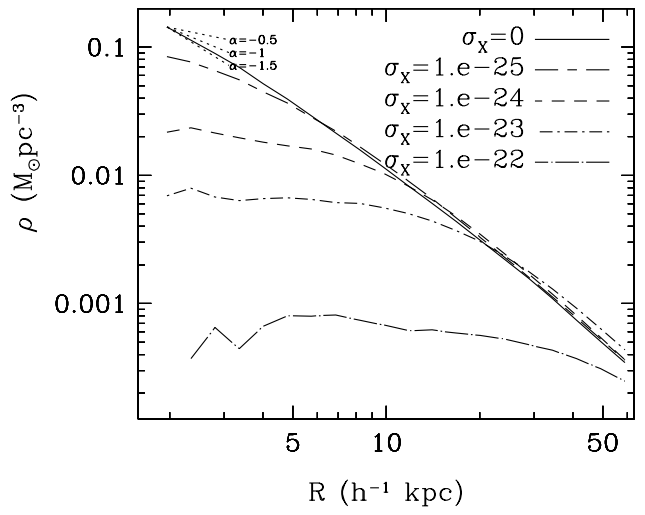

Figure 13: Halo profile of the largest halo in our $64^{3}$ simulations, for a range of $\sigma_{\mathrm{DM}}$ values. Halos are progressively less concentrated and have larger cores with increasing $\sigma_{\mathrm{DM}}$.

In order to explore the high- $\sigma_{\mathrm{DM}}$ limit, we ran $64^{3}$ simulations of SIDM with $\sigma_{\mathrm{DM}}=10^{-25}-10^{-22} \mathrm{~cm}^{2} \mathrm{GeV}^{-1}$. The most illustrative result is to compare the density profile of the largest halo in all our $64^{3}$ simulations, as shown in Figure 13. As seen in Figure 1, there is a smooth trend of increasing core radius with $\sigma_{\mathrm{DM}}$. SIDM with $\sigma_{\mathrm{DM}}=10^{-25} \mathrm{~cm}^{2} \mathrm{GeV}^{-1}$ is quite similar to CDM, though it may also have a core below our $2 h^{-1} \mathrm{kpc}$ resolution limit. Increasing $\sigma_{\mathrm{DM}}$ to $10^{-22} \mathrm{~cm}^{2} \mathrm{GeV}^{-1}$, we continue to see no evidence for the development of an isothermal core due to accelerated heat transfer. The reason is because the collisions are so frequent in the outer portion of the halo that a dense core cannot develop. Instead, collisions randomize the dark matter velocities and prevent a smooth radial inflow required to generate a dense core. As dynamically hot material accretes onto the halo, heat keeps flowing inward and a large core is maintained. Our results are in better agreement with Bryan as opposed to Moore et al. (2000) and Yoshida et al. (2000a). This also illustrates why simulating SIDM beginning with an isolated cuspy Hernquist profile may not be appropriate for large $\sigma_{\mathrm{DM}}$; one should at least begin with a halo profile that is self-consistently stable for a few dynamic times.

\section{SUMMARY}

We present a set of cosmological self-interacting dark matter simulations having cross-sections in the range favored by Spergel \& Steinhardt (2000). Our simulations include the growth of halos from linear fluctuations in a random volume of the universe, with sufficient volume and resolution to obtain a statistical sample of galactic halos resolved to $1 h^{-1} \mathrm{kpc}$. We compare the resulting halos on a case-by-case basis to those in a collisionless CDM simulation having the same initial conditions.

Overall, SIDM is remarkably successful at reproducing observations of the inner portions of dark matter halos where CDM appears to fail. In particular, we find:

1. The inner slopes of SIDM with $\sigma_{\mathrm{DM}}=$ $10^{-23} \mathrm{~cm}^{2} \mathrm{GeV}^{-1}$ typical halos have $\alpha \approx-0.4$ at $r \sim 1 h^{-1} \mathrm{kpc}$, with some scatter in $\alpha$. Our CDM halos have $\alpha \approx-1.5$, in agreement with previous studies (e.g. Moore et al. 1999). SIDM with $\sigma_{\mathrm{DM}}=10^{-24} \mathrm{~cm}^{2} \mathrm{GeV}^{-1}$ is intermediate between these cases, with median $\alpha \approx-0.9$. SIDM is in better agreement with a preliminary analysis of $\mathrm{H} \alpha$ rotation curves of low surface brightness galaxies (Dalcanton \& Bernstein 2000).

2. SIDM with $\sigma_{\mathrm{DM}}=10^{-23} \mathrm{~cm}^{2} \mathrm{GeV}^{-1}$ produces central densities $\rho_{c} \sim 0.01 M_{\odot} \mathrm{pc}^{-3}$ at $1 h^{-1} \mathrm{kpc}$, and shows no trend with halo mass. SIDM with $\sigma_{\mathrm{DM}}=$ $10^{-24} \mathrm{~cm}^{2} \mathrm{GeV}^{-1}$ has somewhat higher $\rho_{c}$ values, but remains fairly independent of mass. Conversely, $\rho_{c}$ in CDM halos is much larger than observed, typically $\gtrsim 0.1 M_{\odot} \mathrm{pc}^{-3}$ at $1 h^{-1} \mathrm{kpc}$, and shows a strong trend with halo mass. With their steep profiles, CDM halos are in significantly worse agreement at smaller radii. SIDM is thus is in better agreement with observations, as has also been argued by Firmani et al. (2000a).

3. Simulations with SIDM having $\sigma_{\mathrm{DM}}=$ $10^{-24} \mathrm{~cm}^{2} \mathrm{GeV}^{-1}$ are intermediate between CDM and SIDM with $\sigma_{\mathrm{DM}}=10^{-23} \mathrm{~cm}^{2} \mathrm{GeV}^{-1}$, indicating a smooth increase in the effect of SIDM with cross section, a result that extends (using lower-resolution simulations) from $\sigma_{\mathrm{DM}}=10^{-25} \rightarrow$ $10^{-22} \mathrm{~cm}^{2} \mathrm{GeV}^{-1}$. In particular, the generation of singular isothermal halos is not seen in any of the massive halos simulated, even for $\sigma_{\mathrm{DM}}=$ $10^{-22} \mathrm{~cm}^{2} \mathrm{GeV}^{-1}$. This suggests that the dynamical process of halo growth in a cosmological setting helps keep outer regions of halos hot and prevents core collapse in a Hubble time.

4. We introduce a new mass concentration parameter $c_{M}$ based on a more directly observable quantity, the enclosed mass within tens of kpc. This halo concentration is significantly lower in SIDM models as compared to CDM, providing an observationally accessible discriminant that is not dependent on fitting a particular profile form. A rough estimate of $c_{M}$ for the Milky Way, with large corrections for baryonic effects, favors SIDM over CDM.

5. The central phase space density is lower in SIDM vs. CDM mostly due to the reduction in $\rho_{c}$. The velocity dispersions in the inner regions are quite similar. Both SIDM and CDM are consistent with observations shown in Dalcanton \& Hogan (2000), though SIDM is mildly favored.

6. SIDM produces halos that are more spherical, especially in their inner regions, as compared to CDM. In principle, this is one of the strongest tests of the SIDM paradigm, as near the center any value of $\sigma_{\mathrm{DM}}$ that has a non-negligible effect on the dark matter distribution will increase the core sphericity, while CDM cores are almost always significantly triaxial. 
However, baryons are likely to dominate the shapes of the inner parts of halos, complicating a direct comparison, and in the outer parts the differences between SIDM and CDM are less pronounced.

7. The number of subhalos around our largest (Milky Way-sized) halo is somewhat reduced with increasing $\sigma_{\mathrm{DM}}$, but due to discreteness effects in our Monte Carlo $N$-body technique, we cannot put robust quantitative estimates on the strength of this effect.

Based on these simulations, our currently favored value for $\sigma_{\mathrm{DM}}$ is somewhere between $10^{-23} \mathrm{~cm}^{2} \mathrm{GeV}^{-1}$ and $10^{-24} \mathrm{~cm}^{2} \mathrm{GeV}^{-1}$. Such a cross section simultaneously reproduces both the observed central density and inner slope, as well as being consistent with various observations considered here, which is non-trivial. In contrast, for instance, warm dark matter has difficulty simultaneously reproducing the observed central densities, inner slopes and subhalo population Colin, Avila-Reese \& Valenzuela 2000).

As stated before, inner halo shapes may provide a strong discriminant between CDM and SIDM. On galactic scales, they are difficult to observe and confused by baryonic contributions. Conversely, clusters provide a cleaner test because they have large cores that are not baryondominated, and their mass distributions are directly observable via lensing. Miralda-Escudé (2000) uses the asphericity of cluster MS 2137-23 to (analytically) argue that $\sigma_{\mathrm{DM}}<10^{-25.5} \mathrm{~cm}^{2} \mathrm{GeV}^{-1}$, effectively ruling out SIDM as a solution to halo concentration problems. On the other hand, CL $0024+1654$ is very spherical, much more so than CDM models generally predict (Tyson, Kochanski \& Dell'antonio 1998). Our simulations cannot directly address the shapes of clusters, as we have no cluster-sized objects in our volume. However, SIDM shows some range of halo shapes due to asymmetric infall and unrelaxed mass distributions, so it is unclear whether a single object can definitively rule out SIDM. Support for this statement is provided by Yoshida et al. (2000b), whose cluster has enough triaxiality to be consistent with MS 2137-23 even for $\sigma_{\mathrm{DM}} \approx 2 \times 10^{-24} \mathrm{~cm}^{2} \mathrm{GeV}^{-1}$ (their model S1Wb), contradicting Miralda-Escudé's scaling argument. We note that halo shapes are unaffected by annihilating (Kaplinghat, Knox \& Turner 2000) or decaying (Cen 2000) dark matter, thus they also provide a discriminant between these variants and SIDM.

The cluster core sizes in the simulations of Yoshida et al. (2000b) are larger than observed $\left(\sim 30-70 h^{-1} \mathrm{kpc}\right.$, Miralda-Escudé 1995; Tyson, Kochanski \& Dell'antonio 1998), certainly for $\sigma_{\mathrm{DM}} \approx 2 \times 10^{-23} \mathrm{~cm}^{2} \mathrm{GeV}^{-1}$ $\left(160 h^{-1} \mathrm{kpc}\right)$, and probably even for $\sigma_{\mathrm{DM}} \approx 2 \times$ $10^{-24} \mathrm{~cm}^{2} \mathrm{GeV}^{-1}\left(100 h^{-1} \mathrm{kpc}\right)$. So it may be that SIDM has difficulty matching observations at both dwarf galaxy and cluster scales. However, adiabatic contraction of baryons during the formation of the $\mathrm{cD}$ galaxy will reduce the cluster core radius from $N$-body predictions, so the discrepancy may not be that large. In any case, SIDM with $\sigma_{\mathrm{DM}} \approx 10^{-24}-10^{-23} \mathrm{~cm}^{2} \mathrm{GeV}^{-1}$ comes remarkably close to matching dwarf galaxies, $L^{*}$ galaxies and clusters given the $\sim 10^{5}$ range in mass scales, so we reserve judgement pending a more careful comparison with observations. Yoshida et al. (2000b) mention that SIDM core sizes would be in better agreement with observations if $\sigma_{\mathrm{DM}} \propto v^{-1}$, which would result in the effects of selfinteractions being diminished in hot cluster environments as compared to galaxies. Such a scenario occurs naturally if the dark matter-dark matter scattering has low-lying resonance or bound state contributions, as is the case for ordinary nucleons.

Another theoretical avenue explored in relation to SIDM has been modeling the Tully-Fisher relation. Hydrodynamic simulations show that the simulated Tully-Fisher zero point may be brought into agreement with observations only if halos are less centrally concentrated than predicted by CDM (Navarro \& Steinmetz 2000). Mo \& Mao (2000) determine that a cross section of $\sigma_{\mathrm{DM}} \sim$ $10^{-23} \mathrm{~cm}^{2} \mathrm{GeV}^{-1}$ would produce a correct Tully-Fisher relation for $v_{\text {circ }} \approx 100 \mathrm{~km} / \mathrm{s}$ halos. Thus it is conceivable that the cross section preferred from halo structure constraints may also alleviate the Tully-Fisher discrepancies. Ostriker (2000) suggested that dark matter interactions in the centers of halos naturally produce central black holes with a mass scaling $M_{\mathrm{BH}} \propto \sigma^{5}$, in agreement with observations (Magorrian et al. 1998; Ferrarese \& Merrit 2000 ), and he estimates $\sigma_{\mathrm{DM}} \lesssim 10^{-24} \mathrm{~cm}^{2} \mathrm{GeV}^{-1}$ in order to avoid central black holes that are too large. However, this estimate is based on an $\rho \propto r^{-2}$ profile all the way in to the black hole. If such a dense center never arises because collisions inhibit its formation, then the limits on $\sigma_{\mathrm{DM}}$ are weakened considerably.

Observations of dark matter halos promise to improve significantly in the coming years, particularly constraints on halo core shapes from lensing and on inner profiles and concentrations of halos from $\mathrm{H} \alpha$ rotation curves. If the inner parts of dark matter halos are found to be generically triaxial, this would be the high place of sacrifice for self-interacting dark matter; conversely, spherical halos would provide strong support for this scenario. The main modeling work yet to be is done is an improved examination of the subhalo populations in SIDM, as well as simulations of a larger range of mass scales. The $N$-body Monte Carlo approach has difficulty achieving a large dynamic range due to the stringent Monte Carlo resolution limit (i.e. discreteness effects in the probabalistic description of collisions), therefore a different approach may be necessary.

The SIDM simulations presented here are a first attempt at examining the effect of self-interacting dark matter within the context of a realistic halo formation scenario. The results are quite encouraging that this simple variant of the cold dark matter paradigm will alleviate a wide range of difficulties faced by CDM on galactic scales. We look forward to further investigations and comparisons with observations.

We thank Julianne Dalcanton, Lars Hernquist, Jerry Ostriker, Penny Sackett, Scott Tremaine, Martin White, and Naoki Yoshida for helpful discussions. We thank Greg Bryan for sharing his unpublished results.

RD and DNS are supported by NASA ATP grant NAG5-7066. DNS and BDW are supported by the NASA MAP/MIDEX program. PJS is supported by United States Department of Energy grant DE-FG02-91ER40671. 


\section{REFERENCES}

Avila-Reese, V., Firmani, C. \& Hernandez, X. 1998, ApJ, 505, 37

Bahcall, N., Ostriker, J. P., Perlmutter, S. \& Steinhardt, P. J. 1999, Science, 284, 1481

Blitz, L., Spergel, D. N., Teuben, P. J., Hartmann, D., Burton, W. B. 1999, ApJ, 514, 818

Bullock, J. Kravtsov, A V., Weinberg, D. H. 2000, ApJ, submitted, astro-ph/0002214

Burkert, A. 2 J00, ApJ, in press, astro-ph/0002409

Buote, D. A. \& Canizares, C. R. 1998, MINRAS, 298, 811

Caldwell, R. R., Dave, R. \& Steinhardt, P. J. 1998, Phys. Rev. Lett., 80,1582

Cen, R. 2000, ApJ, submitted

Cón,_P Avila-Bepse, V. \& Valenzuela, O. 2000, ApJ, submitted, astro-ph/0004115

Dalcantan, J. \& Hogan, C. J. 2000, ApJ, submitted, astro$\mathrm{ph} / 0004381$

Dalcanton, J. J. \& Bernstein, R. A. 2000, in "XVth IAP Meeting, Dynamics of Galaxies: From the Early Universe to the Present", eds. F. Combes, G. A. Mamon \& V. Charmandaris

Debattista, V. P. \& Sellwood, J. A. 1998, ApJ, 493, L5

de Blok, W. J. G., McGaugh, S. S. \& van der Hulst, J. M. 1996, MNRAS, 283, 18

Dubinski, J. \& Carlberg, R. G. 1991, ApJ, 378, 496

Ferrarese, L. \& Merritt, D. 2000, ApJ, submitted, astro-ph/0006053

Firmani, C., D'Onghria, E., Chrincarini, . \& AvifaReese. V. 2000. MNRAS, submitted, astro-ph/0005001

Firmani, C., D'Onghia, E., Avila-Reese V. Chencarini, G. \& Hernandez, X. 2000, MNRAS, in press, astro-ph/0002376

Franx, M., van Gorkom, J. M. \& de Zeew

Goodman, J. 2000, ApJ, submitted, astro-ph/0003018

Hannest S \& Scherrer, R. J. 2000, Phys. Rev. D, in press, astro$\mathrm{ph} / 0003046$

Hernquist, L. 1990, ApJ, 356, 359

Hogan, C. J. \& Dalcanton, J. J. 2000, Phys. Rev. D, in press

Hu,W. Barkana B. Gruzinov, A. 2000, Phys.Rev.Lett., submitted, astro-ph/0003365

Jing, Y. P. \& Suto, Y. 2000, ApJ, 529, L69

Kaplinghrat, M., Kmox, L. \& furner, M. 200, astro-phl /005 210

Katz, N., Weinberg D.H., \& Hernquist, L. 1996. ApJS, 105, 19

Klypin, A., Kravtsov, A. V., Valenzuela, O. \& Prada, F. 1999, ApJ, 522,82

Klypin, A. 2000, Lecture at the Summer School "Relativistic Cosmology: Thegry and Observations", Como, Italy, May 2000, astro-ph/0005504

Kochanek, S. \& White, M. 2000, ApJ, submitted, astro$\mathrm{ph} / \mathrm{d} 003483$

Kravtsov, A.V., Klypin, A., Bullock, J. S. \& Primack, J. P. 1998, ApJ, 502, 48

Ma, C.-P. \& Bertschinger, E. 1995, ApJ, 455, 7
Madsen, J. 2000, Phys. Rev. Lett., submitted, astro-ph/0006074

Magorrian, J. et al. 1998, AJ, 115, 2285

Miralda-Escudé, J. 1995, ApJ, 438, 514

Miralda-Escudé, J. 2000, ApJsubmitted, astro-ph/0002050

Mo, H. J. \& Mao, S. 2000, MNRAS, submitted, astro-ph/0002451

Moore, B., Quinn, T., Governato, F., Stadel, J., Lake, G. 1999, MNRAS, 310, 1147

Moore, B., Ghigna, S., Governato, F., Lake, G., Quinn, T., Stadel, J. \& Tozzi, P. 1999, ApJ, 524, L19

Moore, B., Gelato, S Jenkins A Pearce, F. R. \& Quilis, V. 2000, ApJ, submitted, astro-ph/0002308

Navarro, J. F., Frenk, C. S. \& White, S. D. M. 1996, ApJ, 462, 563

Navarro, J. F. \& Steinmetz, M. 2000, ApJ, 528, 607

Narayanan, V., Spergel, D. N., Davé, R. \& Ma, C.-P. 2000, ApJ, submitted

Olling, R. P. \& Merrifield, M. R. 2000, MNRAf, 311, 361

Ostriker, J. P. 2000, Phys. Rev. Lett., in press astro-ph/9912548

Peebles, P. J. E. 2000, ApJ, submitted, astro-ph/0002495

Rauch, M. 1998, ARA\&A, 36, 267

Sackett, P. D., Rix, H.-W., Jarvis, B. J. \& Freeman, K. C. 1994, ApJ, 436, 629

Sackett, P. D. 1999, in "Galaxy Dynamics", eds. D. Merritt, J.A. Sellwood and M. Valluri, p 393 (SF: 1 SP Conf Sories 182)

Sellwood, J. A. 2000, ApJ, submitted, astro-ph/0004352

Somer-Larsen, J. \& Dolgov, A. 2000, ApJ, submitted, astro$\mathrm{ph} / 9912166$

Spergel Steinhardt, P. J. 2000, Phys. Rev. Lett., in press, astro-ph/9909386

Springel, V., Yoshida N \& White, S. D. M. 2000, NewAst, submitted, astro-ph/0003162

Swaters, R. A., Madore, B. F.\& Trewhella, M. 2000, ApJ, 531, L107

Tittley, E. R. M. P. \& Pearce, F. R. 1999, MNRAS, submitted, astro-ph/9911017

Tyson, J. A., Kochanski, G. P. \& Dell'antonio, I. P. 1998, ApJ, 498, L107

van den Bosch, F. C., Robertson, B. E., Dalcanton, J. J., de Blok, W. J. G. 2000, AJ, 119, 1579

an den Bosch, F. C., Swaters, R. A. 2000, AJ, submitted, astro$\mathrm{ph} / 0006048$

peinberg, D. H., Davé, R., Gardner, J. P., Hernquist, L., \& Katz, N. 1999 in "Photometric Redshifts and High Redshift Galaxies", eds. R. Weymann, L. Storrie-Lombardi, M. Sawicki \& R. Brunner (SF: ASP Conf Series)

Wilkinson, M. I. \& Evans, N. W. 1999, MNRAS, 310, 645

Yoshida, N., Springel, V White, S. D. M. \& Tormen, G. 2000, ApJ, submitted, astro-ph/0002362

Yoshida, N.. submitted, astro-ph/0006134 